\title{
CMB Polarization in Theories of Gravitation with Massive Gravitons
}

\author{
Dennis Bessadat, Oswaldo D. Miranda $\ddagger$ \\ INPE - Instituto Nacional de Pesquisas Espaciais - Divisão de Astrofísica, \\ Av.dos Astronautas 1758, São José dos Campos, 12227-010 SP, Brazil
}

\begin{abstract}
.
We study in this paper three different theories of gravitation with massive gravitons - the modified Fierz-Pauli model, Massive Gravity and the bimetric theory proposed by Visser - in linear perturbation theory around a Minkowski and a flat FriedmannRobertson-Walker background. For the transverse-traceless tensor perturbations we show that the three theories give rise to the same dynamical equations, to the same form of the tensor Sachs-Wolfe effect, and consequently to the same form of the Boltzmann equations for the radiative transfer in General Relativity.

We then analyze vector perturbations in these theories and show that they do not give the same results as in the previous case. We first show that vector perturbations in Massive Gravity present the same form as found in General Relativity, whereas in the modified Fierz-Pauli theory the vector gravitational-wave polarization modes $\left(\Psi_{3}\right.$ amplitudes in the Newman-Penrose formalism) do not decay too fast as it happens in the former case. Rather, we show that such $\Psi_{3}$ polarization modes give rise to an unusual vector Sachs-Wolfe effect, leaving a signature in the quadrupole form $Y_{2, \pm 1}(\theta, \varphi)$ on the Cosmic Microwave Background Radiation polarization. We then derive the details for the Thomson scattering of CMB photons for these $\Psi_{3}$ modes, and then construct the correspondent Boltzmann equations. Based upon these results we then qualitatively show that $\Psi_{3}$-mode vector signatures - if they do exist - could clearly be distinguished on the CMB polarization from the usual $\Psi_{4}$ tensor modes.

We also estimate that the graviton mass limit for the vector modes is $m=10^{-66} \mathrm{~g} \sim$ $10^{-29} \mathrm{~cm}^{-1}$, so that vector modes with masses below this limit exhibit the same dynamical evolution as the massless gravitons.

We argue at the end of this paper that CMB polarization experiments can be decisive to test alternative theories of gravitation by measuring CMB polarization in the $E$-mode.
\end{abstract}

PACS numbers: 04.50.+h, 98.80.-k, 95.36.+x, 95.30.Sf

To appear in Classical and Quantum Gravity

$\dagger$ dbessada@das.inpe.br

$\ddagger$ oswaldo@das.inpe.br 


\section{Introduction}

Among the most important basic predictions of the Theory of General Relativity (GR), we know that only one of them has not been yet directly tested: the existence of Gravitational Waves (GWs). The GWs in the GR present two basic features: first, the particle associated with the wave, the graviton, is massless; second, they have only two polarization states. The first feature comes exactly from the weak-field approximation in the space-time metric, yielding a spin-two massless particle field equation; the second feature comes from the gauge invariance of the field equations under local coordinate transformations. These two features are strictly related, since a massless theory allows gauge transformations, and such transformations lead to two polarization states.

However, a metric theory of gravitation, as we shall see, allows up to six polarization modes in general [1]; furthermore, it was shown in [2] that spherical mass-resonant GW detectors might detect up to six polarization modes. These two facts, one theoretical and the other experimental, motivate us to investigate more carefully a general metric theory of gravitation in order to make it possible to predict further features that in the usual GR might, in principle, be lacking.

An immediate generalization of GR may be constructed taking into account some analogies with the basic ideas of Quantum Field Theory (QFT). The simplest models in QFT usually involve free real massless fields with global internal symmetries as, for example, the electromagnetic field; then, to these models we can add further elements like mass, non-abelian internal symmetries, and so forth. Taking into account again the example of the electromagnetic field, the next generalization of this model is the introduction of a mass (via the Higgs mechanism), giving rise to a massive spin-one particle. These two vector models, the massless and the massive ones, are not theoretical conjectures; they give rise to observable particles, the photon and the gauge bosons $W^{ \pm}$ and $Z^{0}$ respectively. Then, since in GR the metric tensor field $g_{\alpha \beta}$ gives rise only to massless particles, the next extension consists in the introduction of massive degrees of freedom into the metric - the so-called "massive gravitons". There are some different effort towards a theory of gravitation with massive gravitons; in this paper we shall consider the approaches worked out in the references [3], [4], [5], [6] and [7].

The approach followed in references [3] and [4] is an improvement of an earlier model pushed forward by M. Fierz and W. Pauli in the thirties [8]. It is constructed by adding a mass term to the linearized Einstein-Hilbert action through the prescriptions of field theory, that is, a quadratic term in the fields $h_{\alpha \beta}$ appearing in the weak-field limit. We shall call this model as "modified Fierz-Pauli" henceforth. In this model, GWs have six polarization modes [9], which may introduce interesting features in cosmology as we are going to show in this paper.

The second approach, devised by Matt Visser, [5] is based upon a bimetric theory, first pushed forward by the work of Rosen [10]. In this model the mass term is introduced by a quadratic term depending not only upon a dynamical metric as in GR, but upon a nondynamical background metric as well. De Paula et. al. [11] showed that this theory 
leads to exactly the same dynamical equations as in the modified Fierz-Pauli model in the weak-field approximation, so that they are absolutely equivalent in this limit. They also have shown that GWs in this model also possess six polarization modes as expected [9].

In the theory of Massive Gravity, as introduced in [6], the Lorentz-invariance of the mass lagrangian is broken in the following way: the quadratic term in the metric perturbations is split into components, giving rise to five possible combinations, with each combination having a different coefficient; then, these five different coefficients are interpreted as five mass parameters, each of them being proportional to a common scale $m$. It was also shown in [6] that the Fierz-Pauli model is promptly recovered through a suitable choice of the mass parameters. In [7], the masses for the gravitons are introduced through a very clever analogy with the Higgs mechanism in QFT: the Lorentz invariance is spontaneously broken by a convenient choice of the "vacuum" for the Goldstone fields, which leads to the model devised in [6].

In all the models introduced above the van Dam-Veltmann-Zakharov (vDVZ) discontinuity [12], [13] is absent [4], [5], 6], [7]. In particular, it can be shown that Massive Gravity is absolutely free of ghosts and classical instabilities provided the mass parameters obey some constraints [7].

In addition to the efforts towards a direct observation of GWs (see, e.g., ref. [14]), we might consider, as an excellent alternative for the time being (since the GW detectors have not yet reached the proper sensitivity to make such direct observations), an indirect approach, using the recent work performed on the theory of Cosmic Microwave Background Radiation (CMB) anisotropies and polarization. The CMB spectrum might hide some "tracks" of GWs, or signatures, left by the interaction of the primordial cosmological gravitons with the CMB photons. The next generation of CMB satellites after Planck is expected to measure the CMB polarization to a better degree of accuracy, and it can hopefully shed a light on the investigations of primordial GWs through the measurements of the so-called E and B-polarization modes [15]. This means that CMB polarization measurements might not only be decisive to detect GWs, but it might also shed a light on the nature of the gravity itself; in other words, CMB measurements could be decisive to test alternative theories of gravitation. This is exactly our goal in the present work: the analysis of CMB polarization induced by GWs in a theory of gravitation with massive gravitons.

To this end, the present paper is organized as follows: in Section 2 we review the classification of plane GWs in the Newman-Penrose formalism for an arbitrary theory of gravitation, deducing their six possible polarization modes. In Section 3 we review the three different approaches to include massive gravitons into GR, and also discuss cosmological perturbations in these theories. Then, in Section 4 we review the basics of radiative transfer in the presence of weak gravitational fields, laying the ground for discussing the Sachs-Wolfe effect in a theory of gravitation with massive gravitons in Section 5, and the effect of GW vector longitudinal modes on the Thomson scattering in Section 6. In Section 7 we get together all the results, obtaining the related radiation 
transfer equations (Boltzmann equations) for each polarization mode. At the end of this paper we discuss the obtained results and make the correspondent conclusions.

\section{Polarization States for an arbitrary Metric Theory of Gravitation}

The polarization states for a GW in an arbitrary metric theory of gravitation are given by the independent modes of the Riemann tensor. In order to compute its components in an Lorentz-invariant scheme, it is convenient to introduce, following the pioneering work of Newman and Penrose, [16], the quasiorthonormal complex-null basis $(k, l, m, \bar{m})$, where $k$ and $l$ are real null-vectors and $m$ and $\bar{m}$ are a pair of complex numbers, satisfying the following orthogonality relations:

$$
k \cdot l=0, \quad m \cdot \bar{m}=-1, \quad k \cdot \bar{m}=k \cdot m=l \cdot \bar{m}=l \cdot m=0 .
$$

We follow [1] and choose the following set of null vectors,

$$
\begin{array}{ll}
k=-\frac{1}{\sqrt{2}}(1,0,0,1), \quad l=-\frac{1}{\sqrt{2}}(1,0,0,-1), \\
m=-\frac{1}{\sqrt{2}}(0,1, i, 0), \quad \bar{m}=-\frac{1}{\sqrt{2}}(0,1,-i, 0),
\end{array}
$$

all satisfying (11). With the basis given by (2) - (3) we can split the Riemann tensor into its irreducible parts, namely, the Weyl tensor, whose ten independent components are given by five complex scalars $\left(\Psi_{0}, \Psi_{1}, \Psi_{2}, \Psi_{3}, \Psi_{4}\right)$, the Ricci tensor, whose nine independent components are given by the scalars $\Phi_{00}, \Phi_{01}, \Phi_{02}, \Phi_{10}, \Phi_{20}, \Phi_{11}, \Phi_{12}$, $\Phi_{21}, \Phi_{22}$, and the Ricci scalar $\Lambda$. Throughout this section, we consider only plane GW propagating in the $\hat{\mathbf{z}}$ direction, whose time dependence is given by cos $\omega t$, and we use for the flat Minkowski metric $\eta_{\alpha \beta}=\operatorname{diag}\{+,-,-,-\}$.

In this context, we may prove that the differential and algebraic properties of the Riemann tensor reduce the number of independent components to six [1], given by

i) The Weyl tensor:

$$
\begin{aligned}
& \Psi_{0}=\Psi_{1}=0, \\
& \Psi_{2}=-\frac{1}{6} R_{l k l k}, \\
& \Psi_{3}=-\frac{1}{2} R_{l k l \bar{m}}, \\
& \Psi_{4}=-R_{l \bar{m} l \bar{m}}
\end{aligned}
$$

ii) The Ricci tensor:

$$
\begin{aligned}
\Phi_{00} & =\Phi_{01}=\Phi_{10}=\Phi_{02}=\Phi_{20}=0, \\
\Phi_{22} & =-R_{l m l \bar{m}}, \\
\Phi_{11} & =\frac{3}{2} \Psi_{2}, \\
\Phi_{12} & =\bar{\Phi}_{21}=\bar{\Psi}_{3} ;
\end{aligned}
$$


ii) The Ricci scalar:

$$
\Lambda=-\frac{1}{2} \Psi_{2}
$$

We can reduce, therefore, the number of independent components of the Riemann tensor to the set

$$
\left\{\Psi_{2}, \Psi_{3}, \bar{\Psi}_{3}, \Psi_{4}, \bar{\Psi}_{4}, \Phi_{22}\right\} .
$$

Henceforth, we call (13) Newman-Penrose (NP) amplitudes. They play the role of definite helicity states $s=(0, \pm 1, \pm 2)$ under rotations around the $\mathrm{z}$ axis in a nearly Lorentz coordinate frame. In particular, the two real NP amplitudes $\left(\Psi_{2}, \Phi_{22}\right)$ correspond to the state $s=0$ (which defines the scalar modes), whereas the complex NP amplitudes $\left(\Psi_{3}, \bar{\Psi}_{3}\right)$ correspond to $s= \pm 1$ (vector modes), and $\left(\Psi_{4}, \bar{\Psi}_{4}\right)$ to $s= \pm 2$ (tensor modes). These polarization modes can be represented on the $x-y, y-z$ or $x-z$ plane as can be seen through the effects of a GW on a ring of dust particles (see reference [17] for further details). Now, following [1, it is very useful to introduce the "driving-force matrix" $S$,

$$
S_{i j}(t):=R_{i 0 j 0}(u)
$$

where $t$ is the proper time and $u=t-z / c$ represents a null "retarded time" as measured by an ideal detector in the coordinate system $\left\{t, x^{i}\right\}$. From (14) we define a basis for the GW polarizations as follows: first, we represent the NP amplitudes as

$$
\begin{aligned}
& p_{1}(\hat{z}, t)=\Psi_{2}(u), \quad p_{2}(\hat{z}, t)=\operatorname{Re} \Psi_{3}(u), \quad p_{3}(\hat{z}, t)=\operatorname{Im} \Psi_{3}(u), \\
& p_{4}(\hat{z}, t)=\operatorname{Re} \Psi_{4}(u), \quad p_{5}(\hat{z}, t)=\operatorname{Im} \Psi_{4}(u), \quad p_{6}(\hat{z}, t)=\Phi_{22}(u) ;
\end{aligned}
$$

(from now on, whenever the polarization index $r$ appears, it will always indicate a given NP amplitude according to the sequence given in (15, 16), so that $r=1$ stands for $\Psi_{2}$, and so forth). Now, writing the NP amplitudes (5), (6), (17) and (9) in Cartesian coordinates (recall that the NP basis given by (2) - (3) can be written in terms of the coordinates $\left.\left\{t, x^{i}\right\}\right)$, we get the following result

$$
\begin{aligned}
S & =\left(\begin{array}{ccc}
-\frac{1}{2}\left(p_{4}+p_{6}\right) & \frac{1}{2} p_{5} & -2 p_{2} \\
\frac{1}{2} p_{5} & \frac{1}{2}\left(p_{4}-p_{6}\right) & 2 p_{3} \\
-2 p_{2} & 2 p_{3} & -6 p_{1}
\end{array}\right) \\
& =\sum_{r=1}^{6} p_{r}(\hat{z}, t) E_{r}(\hat{z}),
\end{aligned}
$$

where $E_{r}(\hat{z})$ are the basis polarization matrices, given by

$$
\begin{aligned}
& E_{1}=-6\left(\begin{array}{lll}
0 & 0 & 0 \\
0 & 0 & 0 \\
0 & 0 & 1
\end{array}\right), E_{2}=-2\left(\begin{array}{lll}
0 & 0 & 1 \\
0 & 0 & 0 \\
1 & 0 & 0
\end{array}\right) \\
& E_{3}=2\left(\begin{array}{lll}
0 & 0 & 0 \\
0 & 0 & 1 \\
0 & 1 & 0
\end{array}\right), \quad E_{4}=-\frac{1}{2}\left(\begin{array}{rrr}
1 & 0 & 0 \\
0 & -1 & 0 \\
0 & 0 & 0
\end{array}\right),
\end{aligned}
$$




$$
E_{5}=\frac{1}{2}\left(\begin{array}{lll}
0 & 1 & 0 \\
1 & 0 & 0 \\
0 & 0 & 0
\end{array}\right), \quad E_{6}=-\frac{1}{2}\left(\begin{array}{lll}
1 & 0 & 0 \\
0 & 1 & 0 \\
0 & 0 & 0
\end{array}\right) .
$$

Therefore, the polarization of a GW in an arbitrary metric theory of gravity can be fully described by the basis polarization matrices $E_{r}(\hat{z})$. However, due to the tensorial character of the space-time metric it is convenient to cast the polarization basis (19) into a tensor; hence, along with its spatial components, given by $\left(E_{r}\right)_{i j}(\hat{z})$, there are the 00 and $0 i$ components, which are zero by the very definition of the "full driving-force matrix" $S(\underline{14})$, so that

$$
S_{00}(t)=R_{0000}(u)=0, \quad S_{0 i}(t)=R_{0 i 00}(u)=0 .
$$

Hence, the polarization tensor assumes the form

$$
\begin{aligned}
& \varepsilon^{1}=\left(\begin{array}{llll}
0 & 0 & 0 & 0 \\
0 & 0 & 0 & 0 \\
0 & 0 & 0 & 0 \\
0 & 0 & 0 & 1
\end{array}\right), \quad \varepsilon^{2}=\left(\begin{array}{llll}
0 & 0 & 0 & 0 \\
0 & 0 & 0 & 1 \\
0 & 0 & 0 & 0 \\
0 & 1 & 0 & 0
\end{array}\right) \\
& \varepsilon^{3}=\left(\begin{array}{llll}
0 & 0 & 0 & 0 \\
0 & 0 & 0 & 0 \\
0 & 0 & 0 & 1 \\
0 & 0 & 1 & 0
\end{array}\right), \quad \varepsilon^{4}=\left(\begin{array}{rrrr}
0 & 0 & 0 & 0 \\
0 & 1 & 0 & 0 \\
0 & 0 & -1 & 0 \\
0 & 0 & 0 & 0
\end{array}\right), \\
& \varepsilon^{5}=\left(\begin{array}{llll}
0 & 0 & 0 & 0 \\
0 & 0 & 1 & 0 \\
0 & 1 & 0 & 0 \\
0 & 0 & 0 & 0
\end{array}\right), \quad \varepsilon^{6}=\left(\begin{array}{rrrr}
0 & 0 & 0 & 0 \\
0 & 1 & 0 & 0 \\
0 & 0 & 1 & 0 \\
0 & 0 & 0 & 0
\end{array}\right) .
\end{aligned}
$$

\section{The Theory of Gravitation with Massive Gravitons}

\subsection{The modified Fierz-Pauli model}

Let us now analyze how do GWs arise in the case of the Fierz-Pauli modified model. In this case, the graviton mass lagrangian appears as a quadratic term in the perturbation of the metric tensor $h_{\alpha \beta}$ in the weak-field limit, so that its action is given by [3], [4]:

$$
\begin{aligned}
S & =\frac{M_{P l}^{2}}{8} \int d^{4} x\left[h_{\alpha \beta, \gamma} h^{\alpha \beta, \gamma}-2 h_{\alpha \beta}{ }^{\beta} h_{, \gamma}^{\alpha \gamma}+2 h_{\alpha \beta}{ }^{, \beta} h^{, \alpha}-h^{, \alpha} h_{, \alpha}\right. \\
& \left.-4 M_{P l}^{-2} h_{\alpha \beta} T^{\alpha \beta}-m^{2}\left(h_{\alpha \beta} h^{\alpha \beta}-\frac{1}{2} h^{2}\right)\right],
\end{aligned}
$$

where $h$ is given by

$$
h=\eta_{\alpha \beta} h^{\alpha \beta},
$$

and $M_{P l}$ is the Planck mass. If instead of the contribution $m^{2} h^{2} / 2$ to the last term on the right-hand side of (22) one had $m^{2} h^{2}$, this model would correspond to the original Fierz-Pauli action, which is plagued by the vDVZ discontinuity [4]. 
The Einstein equations associated with the action (22) are given by

$$
\begin{aligned}
\partial^{\mu} \partial_{\mu} h_{\alpha \beta} & -h_{\alpha}{ }^{\gamma}{ }_{, \gamma \beta}-h_{\beta}{ }_{, \gamma \alpha}+h_{, \alpha \beta}+\eta_{\alpha \beta} h^{\gamma \delta}{ }_{, \gamma \delta} \\
& \quad-\eta_{\alpha \beta} \partial^{\mu} \partial_{\mu} h+m^{2}\left(h_{\alpha \beta}-\frac{1}{2} \eta_{\alpha \beta} h\right)=-2 M_{P l}^{-2} T_{\alpha \beta} ;
\end{aligned}
$$

then, imposing the conservation of the stress energy-momentum tensor, $\nabla_{\alpha} T^{\alpha \beta}=0$, we get the following constraint to the field $h_{\alpha \beta}$ on a Minkowski background,

$$
\partial_{\alpha} \bar{h}^{\alpha \beta}=0
$$

where we have defined

$$
\bar{h}_{\alpha \beta}=h_{\alpha \beta}-\frac{1}{2} \eta_{\alpha \beta} h
$$

The equation (25) is exactly the same found in GR, but in the present case it emerges as a constraint from the conservation of the stress energy-momentum tensor rather than a gauge choice, as in GR. This constraint eliminates four degrees of freedom out of the ten independent components of the space-time metric, leaving then only six independent modes. Since these modes correspond exactly to the polarization states of the GW, we may readily associate the components of $\bar{h}_{\alpha \beta}$ with the correspondent ones of (21), so that the only nonzero contributions are the spatial components $\bar{h}_{i j}$.

Using the arguments above and plugging equation (25) into (24), we obtain, in the absence of sources,

$$
\left(\partial^{\mu} \partial_{\mu}+m^{2}\right) \bar{h}_{i j}=0
$$

which is clearly a Klein-Gordon equation for a wave propagating in the direction $\hat{\mathbf{k}}=\hat{z}$. For the sake of simplicity we henceforth drop the bar over the tensor on the left-hand side of (26) and simply write it as $h_{i j}$.

Due to the oscillatory character of equation (27) we may expand the tensor field $h_{i j}$ into the Fourier modes as follows,

$$
h_{i j}(x)=\int_{-\infty}^{\infty} \frac{d^{3} k}{(2 \pi)^{3 / 2}} \tilde{h}_{i j}(k) e^{-i k x},
$$

which enables us to write down the following decomposition in terms of the polarization tensor (21):

$$
\tilde{h}_{i j}(k)=\sum_{r=1}^{6} \varepsilon_{i j}^{r}(k) \tilde{h}^{r}(k) .
$$

In particular, for the transverse-traceless (TT) component of the tensor perturbation to the metric $h_{i j}$ (corresponding to the $\Psi_{4}$ mode with $r=4,5$ ), we write

$$
\tilde{h}_{i j}^{\perp}=\varepsilon_{i j}^{4}(k) \tilde{h}^{4}(k)+\varepsilon_{i j}^{5}(k) \tilde{h}^{5}(k)
$$

so that Fourier transforming (30) to get $h_{i j}^{\perp}$ in the configuration space, we see that this tensor satisfies (27), that is

$$
\left(\partial^{\mu} \partial_{\mu}+m^{2}\right) h_{i j}^{\perp}=0
$$


which reduces to the GW equation for GR in the limit $m=0$. The tensor $h_{i j}^{\perp}$ encompasses then both transverse polarization modes "+" and " $\times$ " characteristic of GR.

We write the extension of definition (30) to the $\Psi_{3}$ modes (associated with $r=2,3$ ) as

$$
\tilde{h}_{i j}^{\|}=\varepsilon_{i j}^{2}(k) \tilde{h}^{2}(k)+\varepsilon_{i j}^{3}(k) \tilde{h}^{3}(k)
$$

which corresponds to a longitudinal polarization state. As we shall see in Section 5, these modes can also induce an unusual angular pattern for the CMB photons and, in consequence, induce a different signature in its polarization pattern.

In this paper we do not consider the GW scalar polarization modes $\Psi_{2}$ and $\Phi_{22}$, since they couple to the $\delta g_{00}$ scalar component in the metric perturbation on cosmological scales, and then do not produce "handedness" to excite the CMB B-polarization mode.

\subsection{The bimetric model}

The bimetric model proposed by Visser in [5] combines into a single theory a dynamical space-time metric with a nondynamical metric which allows the introduction of a mass for the graviton even in the strong-field limit. However, as was shown by De Paula et. al. [11], in the weak-field limit the bimetric model is absolutely equivalent to the modified Fierz-Pauli model as discussed above. Due to this property we henceforth consider the modified Fierz-Pauli solely.

\subsection{Massive Gravity}

In the case of Massive Gravity, as we have pointed out in the Introduction, the underlying ideas are a bit different. Let us now write down the space-time metric in the weak-field limit as

$$
g_{\alpha \beta}=\eta_{\alpha \beta}+\delta g_{\alpha \beta}
$$

where $\delta g_{\alpha \beta}$ plays the role of a perturbation to the Minkowski background metric, and assumes the form [6],

$$
\begin{aligned}
& \delta g_{00}=2 \varphi, \quad \delta g_{0 i}=S_{i}-\partial_{i} B, \\
& \delta g_{i j}=-\chi_{i j}-\partial_{i} F_{j}-\partial_{j} F_{i}+2\left(\psi \delta_{i j}-\partial_{i} \partial_{j} E\right),
\end{aligned}
$$

where $\varphi, \psi, B, E$ are scalar fields, $F_{i}$ and $S_{i}$ are vector fields, and $\chi_{i j}$ is a tensor field. The vector and tensor fields of (34) satisfy the well known constraints

$$
\chi_{i j}{ }^{j}=0, \quad \chi_{i}^{i}=0, \quad F^{i}{ }_{i}=S^{i}{ }_{, i}=0
$$

necessary to match the number of independent fields to the ten independent components

of the metric $\delta g_{\alpha \beta}$. Now, we construct the mass contribution to the action of the theory as a quadratic term in the tensor fields as well, but breaking the Lorenz invariance as suggested in 6]

$$
\mathcal{L}_{m}=\frac{M_{P l}^{2}}{2}\left[m_{0}^{2} \delta g_{00}^{2}+2 m_{1}^{2} \delta g_{0 i}^{2}-m_{2}^{2} \delta g_{i j}^{2}+m_{3}^{2} \delta g_{i i} \delta g_{j j}-2 m_{4}^{2} \delta g_{00} \delta g_{i i}\right] .
$$


Now, plugging the decomposition (34) into the mass lagrangian (36) and in the usual Einstein-Hilbert lagrangian of GR, and adding up these terms, we get the following equation for the tensor field $\chi_{i j}[6]$

$$
\left(\partial^{\mu} \partial_{\mu}+m_{2}^{2}\right) \chi_{i j}=0
$$

which is exactly the same equation for the TT metric contribution of the modified Fierz-Pauli model given by (31), representing the "genuine" GW polarization modes for Massive Gravity. As we have argued in Section 3.1, the $\Psi_{3}$ content of the modified Fierz-Pauli model plays the role of extra GW longitudinal polarization modes; however, in Massive Gravity, the vector fields $F_{i}$ and $S_{i}$ evolve as massive spin-one particles with transverse polarization, and have nothing to do with extra GW polarization states.

Besides this original approach devised by V. Rubakov [6], there is another way of breaking the Lorentz symmetry of the massive lagrangian which resembles the Higgs mechanism in QFT. In this case, we introduce a set of four Goldstone fields $\phi^{0}(x)$ and $\phi^{i}(x), i=1,2,3$, and a scalar, a vector and a tensor field constructed as follows [7], [18]:

$$
\begin{aligned}
& X=\Lambda^{-4} g^{\alpha \beta} \partial_{\alpha} \phi^{0} \partial_{\beta} \phi^{0}, \\
& V^{i}=\Lambda^{-4} g^{\alpha \beta} \partial_{\alpha} \phi^{0} \partial_{\beta} \phi^{i}, \\
& W^{i j}=\Lambda^{-4} g^{\alpha \beta} \partial_{\alpha} \phi^{i} \partial_{\beta} \phi^{j}-X^{-1} V^{i} V^{j},
\end{aligned}
$$

where $\Lambda$ is the energy scale of the theory. With such elements we introduce an arbitrary function $F=F\left(X, V^{i}, W^{i j}\right)$, so that the action for Massive Gravity reads

$$
S=\int d^{4} x \sqrt{-g}\left[-M_{P l}^{2} R+\Lambda^{4} F\left(X, V^{i}, W^{i j}\right)+\mathcal{L}_{\text {matter }}\right],
$$

where the first term on the right-hand side represents the usual Einstein-Hilbert action, and $\mathcal{L}_{\text {matter }}$ is the lagrangian for ordinary matter minimally coupled to the metric. We can show that in the linear perturbation regime given by (33), after setting the Goldstone fields to their "vacuum" values,

$$
g_{\alpha \beta}=\eta_{\alpha \beta}, \quad \phi^{0}=\Lambda^{2} t, \quad \phi^{i}=\Lambda^{2} x^{i},
$$

and substituting these values and the decomposition (34) into (41) we get exactly the same mass lagrangian as (36), with the mass parameters now being related to the functions $F$ and their derivatives [7], [18].

\subsection{Cosmological Perturbations for Massive Gravitons}

Once we have discussed the key features of metric perturbations around a flat Minkowski background, let us now address the same question in a flat Friedmann-Robertson-Walker (FRW) background. We start with GR in which, in the standard theory of cosmological perturbations [19], the metric $\delta g_{\alpha \beta}$ is decomposed exactly in the same way as we did in (34) but, in this case, we multiply all these components by the square of the scale factor of the universe $a(\eta)$ ( $\eta$ is the conformal time), that is

$$
\delta g_{\alpha \beta}=a(\eta)^{2}\left(\begin{array}{cc}
2 \varphi & S_{i}-\partial_{i} B \\
S_{i}-\partial_{i} B & -\chi_{i j}-\partial_{i} F_{j}-\partial_{j} F_{i}+2 \psi \delta_{i j}-2 \partial_{i} \partial_{j} E
\end{array}\right) .
$$


The constraints for the cosmological case are the same as (35). Now, since we are interested in the CMB polarization induced by GWs, we focus only on the TT part of the metric perturbation; in this case, the Einstein equation for the tensor field is given by (the prime' indicates a derivative with respect to conformal time)

$$
h^{\prime \prime}{ }_{i j}-\nabla^{2} h_{i j}+2 \mathcal{H} h_{i j}^{\prime}=0,
$$

which simply describes transverse GW travelling on an expanding background.

In the case of the modified Fierz-Pauli model the same metric decomposition cannot be performed due to the extra polarization modes; we instead introduce

$$
\delta g_{\alpha \beta}=a(\eta)^{2}\left(\begin{array}{cc}
2 \phi & X_{i}-Q_{, i} \\
X_{i}-Q_{, i} & -h_{i j}
\end{array}\right),
$$

where $\phi$ and $Q$ are scalar fields, $X_{i}$ is a divergenceless vector field, and $h_{i j}$ is the cosmological version of the tensor given by the solution to equation (27), carrying the correspondent six polarization modes spanned in the NP formalism. The two scalar fields, plus the two components of the transverse vector field and the six modes of the tensor field give exactly the required ten degrees of freedom. The mass lagrangian for this model can be constructed analogously as in (22), that is, it appears as a quadratic term in the metric (45). The full action is then obtained by adding up this contribution to the usual Einstein-Hilbert one, and the Einstein equations can be derived using the standard tools. Before doing that, it is convenient to decompose the tensor perturbation $h_{i j}$ into its TT and longitudinal parts in the Fourier space. In (28) the whole time-dependence of $h_{i j}$ is contained in the exponential since it is a solution to a wave equation of the form (27); now, such time-dependence changes because of the extra temporal function $a(\eta)$ appearing in (45), which introduces a damping in the oscillation. Therefore, we Fourier-expand the massive tensor perturbation $h_{i j}$ as

$$
h_{i j}(x)=\int_{-\infty}^{\infty} \frac{d^{3} \mathbf{k}}{(2 \pi)^{3 / 2}} \tilde{h}_{i j}(\eta, \mathbf{k}) e^{-i \mathbf{k} \cdot \mathbf{r}},
$$

where

$$
\tilde{h}_{i j}(\eta, \mathbf{k})=\sum_{r=1}^{6} \varepsilon_{i j}^{r}(\mathbf{k}) \tilde{h}^{r}(\eta, \mathbf{k}),
$$

so that the TT and longitudinal components of $\tilde{h}_{i j}$ can be written in the same foot as (30) and (32), that is

$$
\begin{aligned}
& \tilde{h}_{i j}^{\perp}(\eta, \mathbf{k})=\varepsilon_{i j}^{4}(\mathbf{k}) \tilde{h}^{4}(\eta, \mathbf{k})+\varepsilon_{i j}^{5}(\mathbf{k}) \tilde{h}^{5}(\eta, \mathbf{k}), \\
& \tilde{h}_{i j}^{\|}(\eta, \mathbf{k})=\varepsilon_{i j}^{2}(k) \tilde{h}^{2}(\eta, \mathbf{k})+\varepsilon_{i j}^{3}(\mathbf{k}) \tilde{h}^{3}(\eta, \mathbf{k}) ;
\end{aligned}
$$

now, extracting the Einstein equations from the action for the cosmological Fierz-Pauli model as we have sketched above, we see that both fields $h_{i j}^{\perp}$ and $h_{i j}^{\|}$satisfy the same dynamical equations

$$
\begin{aligned}
& h^{\perp^{\prime \prime}}{ }_{i j}-\nabla^{2} h^{\perp}{ }_{i j}+2 \mathcal{H} h_{i j}^{\perp^{\prime}}+a^{2} m^{2} h_{i j}^{\perp}=0, \\
& h^{\|^{\prime \prime}}{ }_{i j}-\nabla^{2} h^{\|}{ }_{i j}+2 \mathcal{H} h_{i j}^{\|^{\prime}}+a^{2} m^{2} h_{i j}^{\|}=0 .
\end{aligned}
$$


In the case of Massive Gravity, the cosmological perturbations to the metric are given by (43), together the following set of perturbations to the Goldstone fields in the unitary gauge (42),

$$
\tilde{\phi}^{0}=\phi^{0}+\Lambda^{2} \lambda^{0}, \quad \tilde{\phi}^{i}=\phi^{i}+\Lambda^{2}\left(\lambda^{i}+\lambda^{, i}\right),
$$

where $\lambda^{0}$ e $\lambda$ are scalar fields and $\lambda^{i}$ is a divergenceless vector field. Now, under infinitesimal coordinate transformations

$$
\tilde{\eta}=\eta+\xi^{0}, \quad \tilde{x}^{i}=x^{i}+\xi^{i}
$$

we can show that the following vector fields

$$
\varpi_{i}=S_{i}+F_{i}^{\prime}, \quad \sigma_{i}=\lambda_{i}-F_{i},
$$

are invariant.

The action for Massive Gravity on a flat FRW background is then given by (41) with (43) and the Goldstone fields set to their vacuum values (42); the matter lagrangian $\mathcal{L}_{\text {matter }}$ is assumed to be described by a perfect fluid whose perturbations for the fluid four-velocity are

$$
\delta u_{i}=a\left(\zeta_{i}+\partial_{i} \zeta\right), \quad \delta u_{0}=a \varphi .
$$

With these features, the Einstein equations for the tensor field $\chi_{i j}$ are given by [20],

$$
\chi_{i j}^{\prime \prime}-\nabla^{2} \chi_{i j}+2 \mathcal{H} \chi_{i j}^{\prime}+a^{2} m_{2}^{2} \chi_{i j}=0,
$$

whereas for the gauge-invariant vector fields defined by (54) the Einstein equations read

$$
\begin{aligned}
& \nabla^{2} \varpi_{i}-2 a^{2} \rho_{m} M_{p l}^{-2}(1+w) \zeta_{i}=0, \quad \varpi_{i}^{\prime}+2 \mathcal{H} \varpi_{i}-a^{2} m_{2}^{2} \sigma_{i}=0, \\
& m_{2}^{2} \nabla^{2} \sigma_{i}=0,
\end{aligned}
$$

where $\delta_{\zeta}=\zeta-\left(E^{\prime}+B\right)$, and $w$ is the parameter appearing the equation of state of the ordinary matter, $p=w \rho[20]$.

Solving equations (57) - (58) we conclude that the only relevant vector field is $\varpi_{i}$, whose amplitude decays with $a^{-2}$ [20], which is exactly the same behavior of vector fields as derived in GR.

Therefore, the Fierz-Pauli modified model and Massive Gravity give rise to the same results for the TT polarization modes of the tensor perturbations as can be seen from equations (50) and (56), whereas for vector perturbations the situation changes drastically. In the modified Fierz-Pauli model the vector modes of GW polarization obey the same equation as the TT modes, (51), so that they really may contribute to the polarization of CMB as we have shown in [21] for the field $\chi_{i j}$; however, in Massive Gravity, the vector perturbations behave exactly as in GR, which means that they decay too fast after the inflationary phase and do not leave any signature on CMB polarization.

Therefore, the vector modes of the modified Fierz-Pauli model, unlike the predictions of GR, Massive Gravity, and other modified models of gravity, can leave signatures on the CMB. To see how this can be achieved, let us estimate now a limit mass to be detected by CMB polarization experiments, according to the discussion in 
[21]. Since the equations for the tensor (50), and vector (51) modes are identical, the dispersion relations are also identical, given by

$$
\omega^{2}=k^{2}+m^{2}
$$

now, since in GR only GW with frequencies $\nu$ within the range $10^{-15} \mathrm{~Hz}$ to $10^{-18} \mathrm{~Hz}$ may leave a signature on CMB polarization [22], we see that these frequencies correspond to comoving wavenumbers $k$ within the range $10^{-25} \mathrm{~cm}^{-1}$ to $10^{-28} \mathrm{~cm}^{-1}$. We then use the values of $k$ of GR, varying the frequencies in order to obtain constant nonzero graviton masses through equation (59). As a result, if the values of the graviton mass $m$ lie within the range $10^{-66}-10^{-62} \mathrm{~g}$, the correspondent frequencies have values very close to the

expected in GR; in particular, there is a graviton mass limit, $m=10^{-66} \mathrm{~g} \sim 10^{-29} \mathrm{~cm}^{-1}$, so that below this limit the dynamical evolution of the massive modes is indistinguishable from the tensor massless modes. Hence, since the vector modes in the modified FierzPauli model are governed by the same equation as the tensor modes, then the graviton mass limit for the vector modes should be exactly the same.

Along with the value discussed above for the vector massive modes, there are other mass limits obtained so far in the literature by using different tests. For instance, Goldhaber and Nieto [23] found a limit $m<2.0 \times 10^{-62} g$ analyzing the motion of galaxies in clusters. Later on, Talmadge et al. 24] studied the variations of Kepler's third law when compared with the orbits of Earth and Mars, and found a limit $m<7.68 \times 10^{-55} \mathrm{~g}$. Recently, Finn and Sutton [3] calculated the decay of the orbital period of the binary pulsars PSR B1913+16 (Hulse and Taylor pulsar) and PSR B1534+12 due to emission of massive gravitons, and found $m<1.4 \times 10^{-52} \mathrm{~g}$.

\section{The Radiative Transfer Equation in the presence of Weak Gravitational Fields - an overview}

Once we have discussed the key features of the different theories of gravitation with massive gravitons, we now turn our attention to the polarization of CMB. To do so, we initially review the theory of the radiative transfer in the presence of weak gravitational fields, following closely the seminal paper by Polnarev [25] (for a pedagogical introduction, see [26]).

Let us consider a given beam of radiation characterized by its Stokes parameters $\left\{I_{l}, I_{r}, U\right\}$, where $I_{l}$ and $I_{r}$ are the intensities of the radiation in the directions $l$ and $r$, respectively; $I=I_{l}+I_{r}$ is the total intensity of the wave, and the parameter $Q$ is given by $Q=I_{l}-I_{r}$. These functions are strictly related to the photon distribution function, which can be cast in a symbolic vector of the form [27]

$$
\hat{f}:=\left(\begin{array}{c}
I_{l} \\
I_{r} \\
U
\end{array}\right),
$$

where $U$ is the other parameter associated with linear polarization. The components of $\hat{f}$ are functions of the conformal time $\eta$, the comoving spatial coordinates $\mathbf{r}$, and also of 
the photon angular distribution. Since the photons are scattered by the free electrons prior to recombination via Thomson scattering, their distribution function will be shifted according to the equation [25]

$$
\frac{\partial \hat{f}}{\partial \eta}+\frac{p^{i}}{p} \frac{\partial \hat{f}}{\partial x^{i}}+\frac{\partial \hat{f}}{\partial \nu} \frac{d \nu}{d \eta}=C[\hat{f}],
$$

where $\nu$ is the photon frequency, $\hat{\mathbf{p}}$ is the photon momentum, $C[\hat{f}]$ is the scattering term given by

$$
C[\hat{f}]=-\sigma_{T} N_{e} a\left[\hat{f}-\frac{1}{4 \pi} \int_{-1}^{1} d \mu^{\prime} d \varphi P\left(\mu, \varphi, \mu^{\prime}, \varphi^{\prime}\right) \hat{f}\right],
$$

$\sigma_{T}$ is the Thomson scattering cross-section, $N_{e}$ is the number of free electrons in the unit comoving volume, $\mu=\cos \theta$, and $P\left(\mu, \varphi, \mu^{\prime}, \varphi^{\prime}\right)$ is the scattering matrix given by [27]

$$
\begin{aligned}
P\left(\mu, \varphi, \mu^{\prime}, \varphi^{\prime}\right) & =\tilde{P}\left[P^{0}\left(\mu, \mu^{\prime}\right)+\sqrt{1-\mu^{2}} \sqrt{1-\mu^{\prime 2}} P^{1}\left(\mu, \varphi, \mu^{\prime}, \varphi^{\prime}\right)\right. \\
& \left.+P^{2}\left(\mu, \varphi, \mu^{\prime}, \varphi^{\prime}\right)\right]
\end{aligned}
$$

where

$$
\begin{aligned}
\tilde{P} & =\left(\begin{array}{llll}
1 & 0 & 0 & 0 \\
0 & 1 & 0 & 0 \\
0 & 0 & 1 & 0 \\
0 & 0 & 0 & 2
\end{array}\right), P^{0}=\frac{3}{4}\left(\begin{array}{cccc}
2\left(1-\mu^{2}\right)\left(1-\mu^{\prime 2}\right)+\mu^{2} \mu^{\prime 2} & \mu^{2} & 0 & 0 \\
\mu^{\prime 2} & 1 & 0 & 0 \\
0 & 0 & 0 & 0 \\
0 & 0 & 0 & \mu \mu^{\prime}
\end{array}\right), \\
P^{1} & =\frac{3}{4}\left(\begin{array}{cccc}
4 \mu \mu^{\prime} \cos \psi & 0 & -2 \mu \sin \psi & 0 \\
0 & 0 & 0 & 0 \\
2 \mu^{\prime} \sin \psi & 0 & \cos \psi & 0 \\
0 & 0 & 0 & \cos \psi
\end{array}\right), \\
P^{2} & =\frac{3}{4}\left(\begin{array}{cccc}
\mu^{2} \mu^{\prime 2} \cos 2 \psi & -\mu^{2} \cos 2 \psi & -\mu^{2} \mu^{\prime} \sin 2 \psi & 0 \\
-\mu^{\prime 2} \cos 2 \psi & \cos 2 \psi & \mu^{\prime} \sin 2 \psi & 0 \\
\mu \mu^{\prime 2} \sin 2 \psi & -\mu \sin 2 \psi & \mu \mu^{\prime} \cos 2 \psi & 0 \\
0 & 0 & 0 & 0
\end{array}\right)
\end{aligned}
$$

where we have defined $\psi:=\varphi-\varphi^{\prime}$.

In equation (61) the third term on the left-hand side shows the influence or signature of the GWs on the CMB polarization, whereas the term on the right-hand side gives the details of the Thomson scattering. Since the term (62) depends upon the photon angular function through the angles $(\mu, \varphi)$, we see that different GW imprints may lead to different processes of polarization via Thomson scattering. In the next two sections we address these issues in detail. 


\section{The Sachs-Wolfe effect induced by Massive Gravitons}

As we have seen in Section 3.4, the modified Fierz-Pauli model and Massive Gravity are equivalent with respect to the GW TT tensor modes, but only the vector modes of the first model may give rise to relevant contributions to CMB polarization. Hence, from this section on, we shall always consider only the Fierz-Pauli model to address all the issues concerning $\mathrm{GW}$ vector modes.

We have discussed in Section 4 that the CMB photons are polarized due to the Thomson scattering with the free electrons in the epoch of recombination. Prior to Thomson scattering, the cosmological perturbations imprint a signature on the photon angular pattern, the so-called Sachs-Wolfe (SW) effect, which can be understood as the shift of photon frequency along the line of sight.

This effect can be computed through the geodesic equation for the photon; then, using the metric perturbation (45) around a flat FRW space, and the constraint $g_{\alpha \beta} p^{\alpha} p^{\beta}=0$, where $p^{\alpha}$ is the photon four-momentum, the geodesic equation reads for the tensor field $h_{i j}$,

$$
\frac{d \nu}{d \lambda}=-\nu\left[\mathcal{H}+\frac{1}{2} \frac{\partial h_{i j}}{\partial \eta} p^{i} p^{j}\right] \frac{d \eta}{d \lambda}
$$

where $\lambda$ is an affine parameter. The product $\varepsilon_{i j} p^{i} p^{j}$ appearing in (67) may be evaluated as follows: first, since the photon travels along an arbitrary direction $\hat{\mathbf{p}}$, let us construct a reference frame around the GW propagation vector $\hat{\mathbf{k}}$ such that the GW polarization modes assume the simplified form given by (21). To this end we introduce the polarization vectors $\left\{\hat{\varepsilon}_{(1)}^{r}, \hat{\varepsilon}_{(2)}^{r}\right\}$, defined by

$$
\varepsilon_{i j}^{r}=\varepsilon_{(1) i}^{r} \varepsilon_{(1) j}^{r}-\varepsilon_{(2) i}^{r} \varepsilon_{(2) j}^{r},
$$

where $\varepsilon_{i j}^{r}$ is given by (21) for each polarization component $r$, satisfying

$$
\hat{\varepsilon}_{(1)}^{r} \cdot \hat{\varepsilon}_{(2)}^{r}=\hat{\varepsilon}_{(1)}^{r} \cdot \hat{\mathbf{k}}=\hat{\varepsilon}_{(2)}^{r} \cdot \hat{\mathbf{k}}=0 .
$$

The trihedron

$$
\left\{\hat{\varepsilon}_{(1)}^{r}, \hat{\varepsilon}_{(2)}^{r}, \hat{\mathbf{k}}\right\}
$$

is then a reference frame around the GW direction $\hat{\mathbf{k}}$ in which the polarization tensor preserves the simple form (21).

Second, let us express the photon momentum $\mathbf{p}$ in terms of spherical coordinates around (70), so that the following relations hold,

$$
\hat{\mathbf{k}} \cdot \hat{\mathbf{p}}=\cos \theta, \quad \hat{\varepsilon}_{(1)}^{r} \cdot \hat{\mathbf{p}}=\sin \theta \cos \varphi, \quad \hat{\varepsilon}_{(2)}^{r} \cdot \hat{\mathbf{p}}=\sin \theta \sin \varphi,
$$

therefore, using (21) and (71), it follows that

$$
\begin{aligned}
& \varepsilon_{i j}^{2} p^{i} p^{j}=\mu \sqrt{1-\mu^{2}} \cos \varphi \propto Y_{2,+1}(\mu, \varphi), \\
& \varepsilon_{i j}^{3} p^{i} p^{j}=\mu \sqrt{1-\mu^{2}} \sin \varphi \propto Y_{2,-1}(\mu, \varphi), \\
& \varepsilon_{i j}^{4} p^{i} p^{j}=\left(1-\mu^{2}\right) \cos 2 \varphi \propto Y_{2,+2}(\mu, \varphi),
\end{aligned}
$$




$$
\varepsilon_{i j}^{5} p^{i} p^{j}=\left(1-\mu^{2}\right) \sin 2 \varphi \propto Y_{2,-2}(\mu, \varphi) ;
$$

where $Y_{l m}(\mu, \varphi)$ are the usual spherical harmonics. The results (72) - (75) show us that the GW imprint upon the photon angular distribution is in the form of a quadrupole, with $m= \pm 2$ for the $\Psi_{4}$ modes ( $r=4,5$, which coincides with GR), and with $m= \pm 1$ for the $\Psi_{3}$ modes $(r=2,3)$.

Hence, from relations (46), (47), (67) and (72)-(75) we get the following geodesic equations:

i) $\Psi_{3}$ :

$$
\frac{1}{\nu_{0}} \frac{d \nu_{0}}{d \eta} \propto-\frac{\partial h^{2,3}}{\partial \eta} Y_{2, \pm 1}(\mu, \varphi),
$$

ii) $\Psi_{4}$ :

$$
\frac{1}{\nu_{0}} \frac{d \nu_{0}}{d \eta} \propto-\frac{1}{2} \frac{\partial h^{4,5}}{\partial \eta} Y_{2, \pm 2}(\mu, \varphi)
$$

where $\nu_{0}=\nu a(\eta)$.

Then, massive gravitons with the $\Psi_{4}$ polarization modes give rise to the usual tensor SW effect in GR, whereas the $\Psi_{3}$ modes do not yield its well-known vector version in GR (see, for example, the discussion in 28]). This happens because $\Psi_{3}$ modes arise as GW longitudinal states of polarization, and not as a massive vector fields as in GR or Massive Gravity. Anyway, they will leave a different signature on the CMB polarization by means of Thomson scattering, as we discuss in the next section.

\section{The Basis for Thomson Scattering}

We now turn to the derivation of the Thomson scattering term (62) for massive gravitons. Let us consider first that the incident radiation prior to be Thomson scattered is unpolarized, with the angular pattern dictated by the SW effect (76) and (77). In this case, the Stokes vectors for the incident radiation are given by

i) $\Psi_{3}$ :

$$
\hat{a}^{2}=\frac{1}{2} \mu \sqrt{1-\mu^{2}} \cos \varphi \hat{\mathbf{u}}, \quad \hat{a}^{3}=\frac{1}{2} \mu \sqrt{1-\mu^{2}} \sin \varphi \hat{\mathbf{u}},
$$

ii) $\Psi_{4}$ :

$$
\hat{a}^{4}=\frac{1}{2}\left(1-\mu^{2}\right) \cos 2 \varphi \hat{\mathbf{u}}, \quad \hat{a}^{5}=\frac{1}{2}\left(1-\mu^{2}\right) \sin 2 \varphi \hat{\mathbf{u}},
$$

where we have defined

$$
\hat{\mathbf{u}}=\left(\begin{array}{l}
1 \\
1 \\
0
\end{array}\right) .
$$

Now, defining the operator $\hat{P}$ as

$$
\hat{P} \hat{\xi}(\mu, \varphi)=\frac{1}{4 \pi} \int_{-1}^{1} d \mu^{\prime} d \varphi^{\prime} P\left(\mu, \varphi, \mu^{\prime}, \varphi^{\prime}\right) \hat{\xi}\left(\mu^{\prime}, \varphi^{\prime}\right),
$$


where $P$ is the scattering matrix (63), it is straightforward to see, for $\hat{\xi}=\hat{a}^{r}$ $(r=2,3,4,5)$, that

$$
\hat{P} \hat{a}^{r}=p \hat{a}^{r}+q \hat{b}^{r}
$$

where $p$ and $q$ are constants, and $\hat{b}^{r}$ is a basis vector such that

$$
\hat{P} \hat{b}^{r}=p^{\prime} \hat{a}^{r}+q^{\prime} \hat{b}^{r}
$$

where $p^{\prime}$ and $q^{\prime}$ are constants as well. From (178) - (83) we readily see that,

i) $\Psi_{3}:$

$$
\hat{b}^{2}=\frac{1}{2} \sqrt{1-\mu^{2}}\left(\begin{array}{r}
\mu \cos \varphi \\
-\mu \cos \varphi \\
2 \sin \varphi
\end{array}\right), \quad \hat{b}^{3}=\frac{1}{2} \sqrt{1-\mu^{2}}\left(\begin{array}{r}
\mu \sin \varphi \\
-\mu \sin \varphi \\
-2 \cos \varphi
\end{array}\right)
$$

ii) $\Psi_{4}$ :

$$
\hat{b}^{4}=\frac{1}{2}\left[\begin{array}{c}
\left(1+\mu^{2}\right) \cos 2 \varphi \\
-\left(1+\mu^{2}\right) \cos 2 \varphi \\
4 \mu \sin 2 \varphi
\end{array}\right], \quad \hat{b}^{5}=\frac{1}{2}\left[\begin{array}{c}
\left(1+\mu^{2}\right) \sin 2 \varphi \\
-\left(1+\mu^{2}\right) \sin 2 \varphi \\
-4 \mu \cos 2 \varphi
\end{array}\right]
$$

The basis vectors given by (78) and (84), (79) and (85) allows us to factor out the angular dependence of the photon distribution vectors, and they constitute the first-order contribution to $\hat{f}$. The remaining contribution for $\hat{f}$ comes from the $h=0$ solution, that is, in the absence of GWs, in which the photon distribution vector is given by

$$
\hat{f}:=\hat{f}_{0}=f_{0}\left(\nu_{0}\right) \hat{\mathbf{u}}
$$

Now, the linearized photon distribution vector can be written as

$$
\hat{f}^{r} \sim \hat{f}_{0}^{r}+e^{-i \vec{k} \cdot \vec{r}}\left[\alpha^{r}\left(\eta, \mu, \nu_{0}\right) \hat{a}^{r}+\beta^{r}\left(\eta, \mu, \nu_{0}\right) \hat{b}^{r}\right],
$$

where $\alpha^{r}\left(\eta, \mu, \nu_{0}\right)$ and $\beta^{r}\left(\eta, \mu, \nu_{0}\right)$ are functions to be determined as solutions of the Boltzmann's equations (61).

\section{The Complete Boltzmann Equations}

Now, once we have obtained the form of the photon distribution vector (87) for the TT and longitudinal GW mode, we are able to write down the full Boltzmann equations (61). They are given by

i) $\Psi_{3}:$

$$
\begin{aligned}
& \chi^{r \prime}+(q-i k \mu) \chi^{r}=H^{r} \\
& \beta^{r \prime}+(q-i k \mu) \beta^{r}=-\frac{3}{8} q \int_{-1}^{1} d \mu^{\prime}\left[-\chi^{r} \mu^{\prime 2}\left(1-\mu^{\prime 2}\right)\right. \\
& \left.\quad+\beta^{r}\left(-1-\mu^{\prime 2}+2 \mu^{\prime 4}\right)\right],
\end{aligned}
$$

for $r=2,3$, and 
ii) $\Psi_{4}$ :

$$
\begin{aligned}
& \xi^{r \prime}+(q-i k \mu) \xi^{r}=H^{r} \\
& \beta^{r \prime}+(q-i k \mu) \beta^{r}=\frac{3}{16} q \int_{-1}^{1} d \mu^{\prime}\left[\beta^{r}\left(1+\mu^{\prime 2}\right)^{2}-\frac{1}{2} \xi^{r}\left(1-\mu^{\prime 2}\right)^{2}\right],
\end{aligned}
$$

for $r=4,5$. The functions $\xi$ and $\chi$ are defined as

$$
\begin{aligned}
& \xi^{r}=\alpha^{r}+\beta^{r}, \\
& \chi^{r}=\alpha^{r}-\beta^{r},
\end{aligned}
$$

and the scattering rate $q$ is defined as $q=\sigma_{T} N_{e} a$, and finally

$$
H^{r}=\frac{1}{2} \frac{\partial h^{r}(\eta)}{\partial \eta}
$$

The equations (90) and (91) for massive gravitons with $\Psi_{4}$ mode are identical to the correspondent ones in GR; the difference here lies on the Boltzmann equations for the $\Psi_{3}$ modes, (88) and (89), which do not appear in GR. Since the vector and tensor modes satisfy the same dynamical equation, and the mathematical form of the equations (88) and (89) is very different from (90) and (91), it is clear that the vector polarization modes of massive gravitons leave a characteristic signature distinguishable from the tensor one, which could, in principle, be probed by measurements on the CMB $E$ and $B$-modes. Since the experiments in the Planck satellite will improve the WMAP5 results for the $E$-mode, we may expect that such future measurements might decide whether nontrivial GW signatures - as we showed here through equations (88) and (89) for $\Psi_{3^{-}}$ modes - appear or not in the CMB polarization spectrum. In this case, we conclude that $\mathrm{CMB}$ polarization measurements may be decisive to test alternative theories of gravitation - in particular, the massive model as we discussed here.

\section{Conclusions}

We have analyzed in this work three theories of gravitation with massive gravitons, and we have shown that the modified Fierz-Pauli model coincides with Massive Gravity for the TT tensor field in linear perturbation theory around Minkowski and flat FRW backgrounds. We have shown that such tensor perturbations associated with massive gravitons give rise to the usual tensor SW effect, lead to the same Boltzmann equations for these modes in GR.

We have deduced the dynamical equations for the GW vector longitudinal polarization modes ( $\Psi_{3}$-modes) in the modified Fierz-Pauli model and shown that they do not give the same results of Massive Gravity, in which vector perturbations behave like in GR; instead, they give rise, in a cosmological scenario, to a nontrivial SW effect which leaves a vector signature of the quadrupolar form $Y_{2, \pm 1}(\mu, \varphi)$ on the CMB polarization. Also, such massive vector modes possess a mass limit of $m \sim 10^{-29} \mathrm{~cm}^{-1}$ as the tensor modes, so that below this limit the Fierz-Pauli model is absolutely indistinguishable from GR in terms of the dynamical evolution. 
Analyzing the Einstein equations for such $\Psi_{3}$-modes we concluded that these vector signatures could be present at recombination epoch, unlike the vector perturbations in GR and Massive Gravity, which would decay too fast and would not leave any signature on CMB polarization. Therefore, we calculated the new basis for the Thomson scattering for such $\Psi_{3}$-modes, and then deduced the appropriate equation for the radiative transport. Based upon these results we have shown qualitatively that $\Psi_{3}$-mode vector signatures could clearly be distinguished on the CMB polarization from the usual $\Psi_{4}$ tensor modes if the former do exist; hence, we could look for such signatures in the $E$-mode performed by Planck satellite.

In this sense we argued that Planck polarization measurements could be decisive to test alternative theories of gravitation.

\section{Acknowledgments}

DB and ODM thanks Odylio Aguiar, Armando Bernui, Thyrso Villela and Carlos Alexandre Wuensche for very helpful discussions. The authors also thank Professor José A. de Freitas Pacheco for very important discussions and for a critical reading of the manuscript. DB also thanks Professor Mark Kamionkowski for clarifying some important points concerning CMB polarization. DB was financially supported by CAPES, and ODM is partially supported by CNPq (grant 305456/2006-7). 
CMB Polarization in Theories of Gravitation with Massive Gravitons

\section{References}

[1] D. M. Eardley, D. M. Lee and A. P. Lightman. Phys. Rev. D 8, 3308 (1973)

[2] C. Stellati, O. D. Miranda and R. M. Marinho. In preparation.

[3] P. J. Sutton and L. S. Finn. Phys. Rev. D 65, 044022 (2002)

[4] G. Gabadadze and A. Gruzinov. Phys. Rev. D 72 12, 124007 (2005)

[5] M. Visser. Gen. Rel. and Grav. 30, 1717 (1998)

[6] V. Rubakov.: Lorentz-violating graviton masses: getting around ghosts, low strong coupling scale and VDVZ discontinuity. hep-th/0407104 (2004)

[7] S. L. Dubovsky. JHEP 10, 76 (2004)

[8] M. Fierz and W. Pauli. Proc. Roy. Soc. Lond. A, 173 (1939)

[9] D.G. Boulware and S. Deser. Phys. Rev. D 6, 3368 (1972)

[10] N. Rosen. Ann. of Phys. 84, 455 (1973)

[11] W. L. S. de Paula, O. D. Miranda and R. M. Marinho. Class. Quantum Grav. 21, 4595 (2004)

[12] H. van Dam and M. Veltman. Nucl. Phys. B22, 397 (1970)

[13] V.I. Zakharov. JETP Lett. 12, 312 (1970)

[14] B.F. Schutz. Class. Quantum Grav. 16, A131, (1999)

[15] M. Tucci et. al. MNRAS 360, 935 (2005)

[16] E. Newman and R. Penrose. J. Math. Phys 3, 566 (1962)

[17] C. M. Will.: The Confrontation between General Relativity and Experiment. Living Reviews in Relativity 3, 9 http://www.livingreviews.org/lrr-2006-3 (2006)

[18] S. L. Dubovsky, P. G. Tinyakov and I. I. Tkachev. Phys. Rev. D 72, 084011 (2005)

[19] V. F. Mukhanov, H. A. Feldman and R. H. Brandenberger.: Theory of cosmological perturbations. Phys. Rep. 215, 203-333 (1992)

[20] M. V. Bebronne and P. G. Tinyakov. Phys. Rev. D 76, 084011 (2007)

[21] D. Bessada and O. D. Miranda.: Polarization of CMB induced by tensor modes of primordial GWs in Massive Gravity Submitted to Class. Quantum Grav. (2008)

[22] R.R. Caldwell, M. Kamionkowski and L. Wadley, Phys. Rev D 59, 027101, (1998).

[23] A. S. Goldhaber and M. M. Nieto.: M. M. Mass of the graviton. Phys. Rev. D 9, 1119, (1974).

[24] C. Talmadge, J. P. Berthias, R. W. Hellings and E. M. Standish.: Model-independent constraints on possible modifications of newtonian gravity. Phys. Rev. Lett. 61, n. 10, (1988).

[25] A. G. Polnarev. Sov. Astron. 29(6), 307 (1985)

[26] P. Cabella and M. Kamionkowski.: Theory of Cosmic Microwave Background Polarization arXiv: astro-ph/0403392 (2004)

[27] S. Chandrasekhar.: Radiative Transfer, Dover Ed. (1960)

[28] M. Giovannini.: Theoretical Tools for CMB Physics. IJMP D 14, 363 (2005) 